\title{
The impact of restricted diet on brain function using BOLD-fMRI
}

Received: 10 February 2006 / Accepted: 12 April 2006 / Published online: 19 May 2006

(C) Springer-Verlag 2006

\begin{abstract}
We investigated the effect of a restricted diet model on activity in the human motor cortex using functional magnetic resonance imaging (fMRI). Two series of blood oxygenation level-dependent (BOLD)-fMRI measurements were made in healthy subjects performing simple motor tasks using their right hands. The first series was done 5-10 days prior to the restricted diet schedule (controls), and the second series was performed after 25-28 days of restricted diet, in the form of a religious fast (Ramadan). The size and intensity of the activated area in the motor cortex increased during the time of restricted diet versus the controls. We conclude that restricted diet has a significant effect on cerebral activity, as shown by BOLD-fMRI, although the exact relationship between the images and neuronal activity due to the restricted diet is still to be determined.
\end{abstract}

Keywords Restricted diet - Fasting - BOLD - fMRI · Motor cortex $\cdot$ Human

S. Boujraf

Biophysics and MRI Methods Department,

Faculty of Medicine and Pharmacy, Fez, Morocco

N. Benajiba

Nutrition and Food Department, University of Cordoba, Cordoba, Spain

F. Belahsen

Neurology Department, University Hospital of Fez, Fez, Morocco

S. Tizniti

Radiology Department, University Hospital of Fez, Fez, Morocco

L. J. Garey

Centre for Psychiatric Neuroscience, University of Lausanne, Lausanne, Switzerland

L. J. Garey ( $\square)$

Route Suisse 43, 1166 Perroy, Switzerland

E-mail:1.garey@freesurf.ch

Tel.: +41-21-8253115

Fax: +41-21-8253115

\section{Introduction}

Functional magnetic resonance imaging (fMRI) based on blood oxygenation level-dependent (BOLD) contrast mechanisms has become a powerful tool to investigate the functional organisation of human and animal brains and has been used to show functionally activated brain regions as a result of visual stimulation, as well as other paradigms (Ogawa et al.1990, 1992; Belliveau et al. 1991; Blamire et al. 1992; Kwong et al. 1992; Turner et al. 1993; Britsch et al. 1996; Kollias et al. 1996; Wildgruber et al. 1996, 1997; Disbrow et al. 1998; Frahm et al. 2004).

Changes in the oxygenation of blood correlate with changes in neuronal activity (Raichle et al. 1994) and lead to MRI signal changes caused by alterations in the physical properties of the blood. While completely oxygenated haemoglobin is diamagnetic, deoxygenated haemoglobin is paramagnetic (Ogawa et al. 1990; Turner et al. 1991; Buxton et al. 1998). As the relevant properties of brain tissue are similar to those of blood, the magnetic flux density is disrupted in the vicinity of vessels containing deoxygenated blood. Haacke et al. (1997) demonstrated that as a result of differences associated with an increase in oxygenation state it is possible to show signal increases in brain tissue using $\mathrm{T} 2 *$-weighted sequences. This is known as the BOLD effect.

Diet is of major importance for general health, with particular impact on blood pressure, flow and oxygenation, and tissue perfusion (Kim et al. 1993; Gati et al. 1997; Mattson et al. 2002, 2003; McGrath-Hanna et al. 2003). These parameters, which may therefore be influenced by a restricted diet, should affect the BOLD signal, which reflects functional activity of the brain (Ogawa et al. 1990, 1992; Turner et al. 1991; Haacke et al. 1997). Nevertheless, to our knowledge no BOLD-fMRI study has been performed on the impact of nutritional behaviour on human brain function in general and oxygenation of the cortex in particular. In this study, we looked for changes in the BOLD-fMRI signal representing 
neural activity during a period of restricted diet (religious fasting) using a simple motor paradigm.

\section{Methods}

\section{Subjects}

Fourteen healthy male right handed volunteers were recruited for this study, but only six were retained for the final analysis, as several conditions had to be taken into account. To be admitted, volunteers had to have no significant history of neurological pathology or trauma, have a consistent, equilibrated and healthy daily nutritional habit, as well as a generally healthy lifestyle, in terms of regular sleep and rest during the whole period of the study and had to be non-smokers. All participants were briefed on MRI scanner safety and gave consent before taking part.

\section{Diet model}

The restricted diet model used in this study was based on fasting during daytime for one month during the Muslim month of Ramadan. This involved voluntary avoidance of food and drink from sunrise to sunset, meaning a daily fast of some $14 \mathrm{~h}$.

\section{BOLD-fMRI paradigm}

The paradigm consisted of a motor task involving opening and closing the dominant (right) hand at a frequency of $1.5 \mathrm{~Hz}$, according to the scheme shown in Fig. 1. The total time of each scan session was $3 \mathrm{~min}$ in which 3 cycles were acquired each consisting of $30 \mathrm{~s}$ "on" alternating with $30 \mathrm{~s}$ "off". The volunteers were trained outside the scanner to keep exercising the same power level during the course of the motor task. They also rehearsed their task in the scanner before each session. Two BOLD-fMRI scan sessions were performed, the first between the 5th and 10th days preceding the start of the fast and the second between days 25 and 28 of the fasting month. In each session, the same BOLD-fMRI measurement was done twice to control the reproducibility of the required motor task. All sessions were performed between 1530 and 1730 hours. BOLD-sensitive fMRI measurements were carried out on a 1.5 Tesla magnet (Signa, General Electric) using a single shot Gradient
Echo, Echo-Planar Imaging (EPI) sequence. The echo time was $50 \mathrm{~ms}$, the repetition time was $3,750 \mathrm{~ms}$ and the flip angle was $90^{\circ}$. Twenty axial slices covering the whole motor cortex were acquired at a slice thickness of $4 \mathrm{~mm}$ and a planar resolution of $2 \times 2 \mathrm{~mm}^{2}$. The activated area in each case was mainly situated in the dorsal part of the pre-central gyrus, but extending slightly to the post-central area. We refer to the activated area as simply "motor area". Individual differences were compensated by the averaging process for residual signal differences, explained below.

\section{Data post-processing}

All data underwent identical post-processing using Statistical Parametric Mapping (SPM 99; Wellcome Department of Cognitive Neurology, London, UK; http://www.fil.ion.ucl.ac.uk/spm; Friston et al. 1995; Matlab version 6.1, Mathworks Inc., USA). The functional images from each subject were analysed individually. The echo-planar images were realigned using a rigid body transformation to the first volume. A spatial normalisation routine was performed. A box-car convolved with a hemodynamic response function was used. $T$-statistics were calculated for each voxel. Raw data were filtered with a Gaussian filter of $5 \mathrm{~mm}$ FWHM (full width at half maximum) in all directions. Significantly activated areas were shown at a threshold of $P<0.01$.

Individual maximal BOLD signal changes in the motor area were calculated for each subject for each BOLD-fMRI session. Then the differences in maximal BOLD signal in the same area between the pre-fasting and fasting sessions were calculated for each subject. Finally the size of the activated motor area was calculated for both pre-fasting and fasting sessions. Activation maps were calculated and overlaid on anatomical images. A group analysis of the data was performed, and subtraction maps of the averages of both sessions were calculated.

\section{Results}

All individual results showed consistent and significant increase of activity in the motor cortex during fasting. Figure 2 shows the size of the activated motor area expressed in pixels $\left(1\right.$ pixel $\left.=2 \times 2 \mathrm{~mm}^{2}\right)$ during prefasting and fasting. The size of the activated area varied
Fig. 1 Motor paradigm used in both restricted diet (fasting) and normal diet (non-fasting)

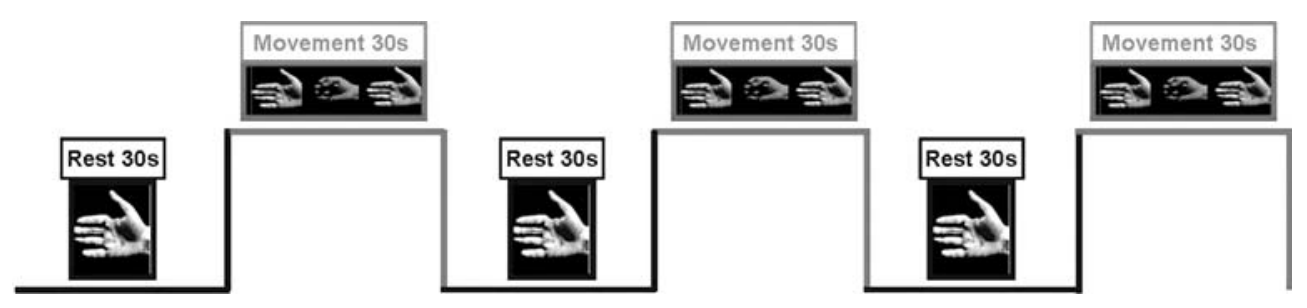


from $49 \pm 25$ to $108 \pm 25$ pixels across the six subjects prior to fasting, while during fasting it was from $114 \pm 16$ to $157 \pm 16$ pixels. The average increase in size was 53 pixels $(P=0.0012)$.

Figure 3 shows the maximal BOLD signal changes in the activated motor area during fasting versus normal diet of the same subjects. All subjects showed an increase of the maximal BOLD signal during the restricted diet protocol, reflecting an increase of activation. The signal in the non-fasting sessions varied from $499 \pm 39$ to $601 \pm 39$ (arbitrary units) over the subjects, while during fasting it was from $513 \pm 43$ to $633 \pm 43$. The average

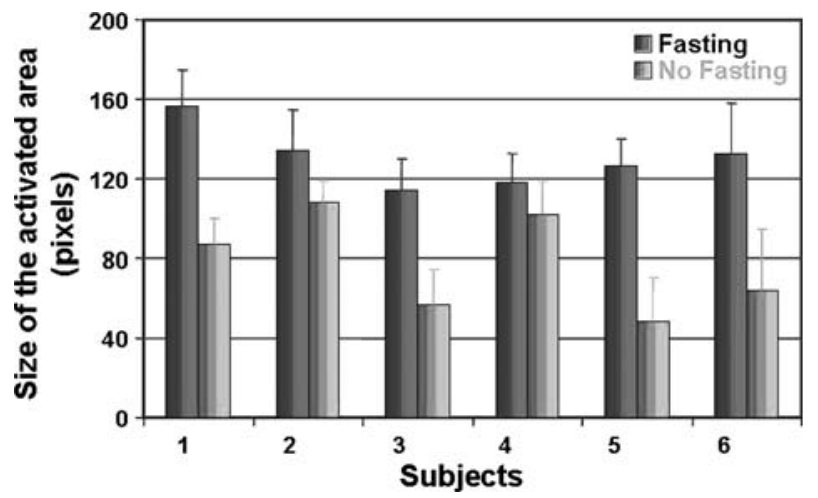

Fig. 2 The size of the activated motor area (in pixels) achieved during restricted diet (fasting) versus normal diet (non-fasting) of each subject. Bars refer to SD increase was $33 \pm 15$. Although non-significant, this signal increase in the activated brain during the restricted diet of from 2.7 to $9.9 \%$ was consistent across subjects. Figure 4 shows the residual BOLD signal changes in the activated motor area. The activation represents the difference between the average maps of all subjects with restricted compared to normal diet. These residual maps reflect the strength of the average activation resulting from the restricted diet versus that from the normal diet protocol.

\section{Discussion}

The size of the activated area in the motor cortex was significantly increased near the end of the fasting month versus a few days prior to the start of the fast $(P<0.01)$. Our results also show a tendency to an increase of the intensity of the maximal BOLD signal reflecting an increase in the oxygenation level of the motor cortex.

Our study suggests that a restricted diet, in this case fasting, can have a marked effect on brain activity. Mattson et al. $(2002,2003)$ suggested that dietary restriction could improve functional deficits in the brain and reduce the risk in major neurodegenerative disorders such as Alzheimer and Parkinson disease. Kennedy and Zochodne (2005) showed that pathological sensory and motor function may recover following spontaneous remission from diabetes with near-euglycaemia. Laijiani et al. (2003) reported a significant decrease in glycaemia
Fig. 3 The maximal BOLD signal changes in the activated motor area during restricted diet (fasting) versus normal diet (non-fasting) of the same subjects, and the percentage signal changes in the activated area during restricted diet compared to normal diet. Bars refer to SD
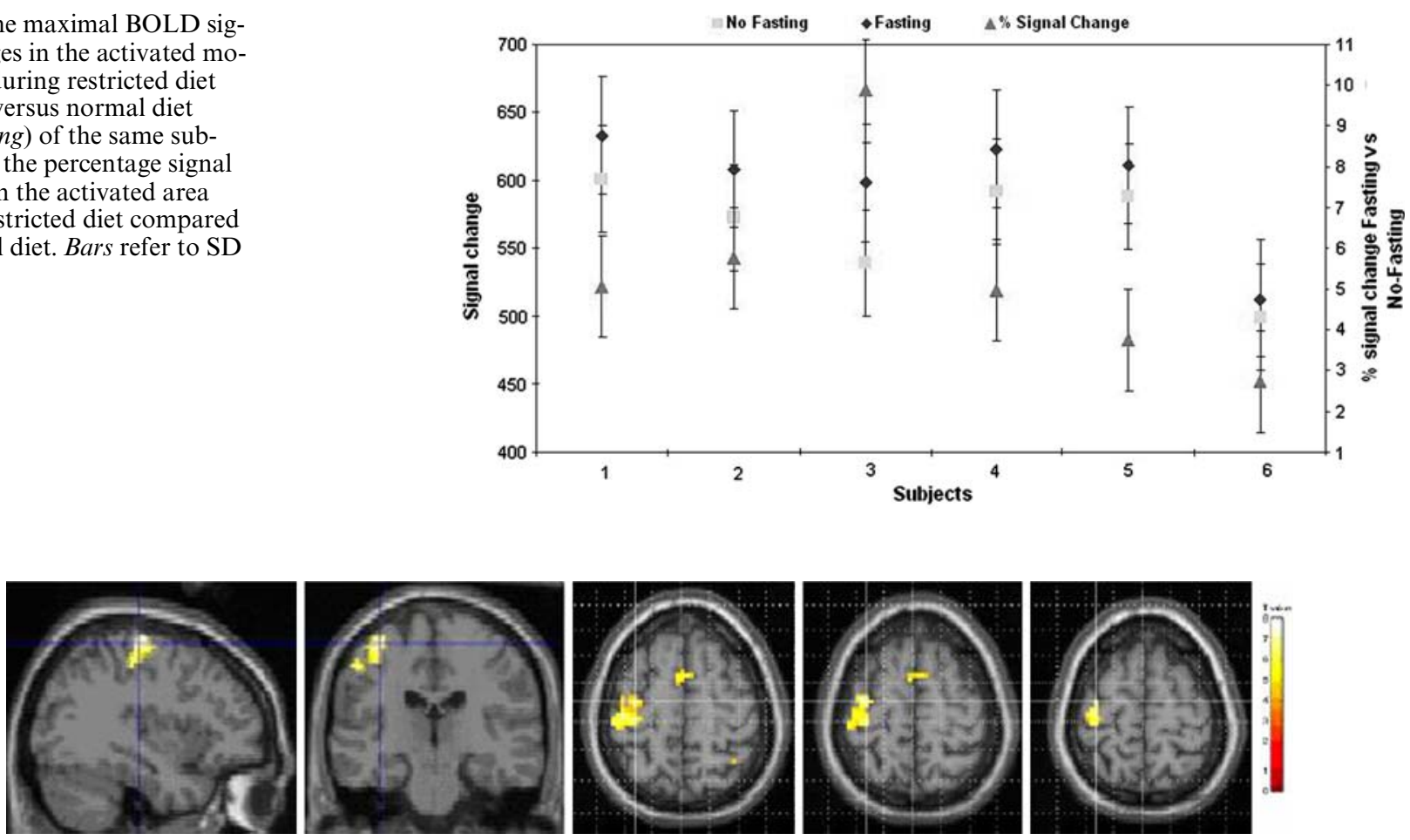

Fig. 4 The residual BOLD signal changes in the activated motor area. The activation shown represents the difference between the average map of all subjects calculated during fasting and non-fasting 
by the end of one month of fasting. However, increased oxygenation and activation of neurons in the motor cortex as recorded by BOLD require energy. In other words, if restricted diet decreases blood glucose concentration, to maintain sufficient oxygenation and energy supply to the brain cerebral blood flow and perfusion would need to be enhanced. Thus restricted diet can have a significant effect on cerebral activity as shown by BOLDfMRI. However, the exact relationship between restricted diet, increased neuronal activity and possible physiological benefit remains to be determined.

\section{References}

Belliveau JW, Kennedy DN, McKinstry RC, Buchbinder BR, Weisskoff RM, Cohen MS, Vevea JM, Brady TJ, Rosen BR (1991) Functional mapping of the human visual cortex by magnetic resonance imaging. Science 254:716-719

Blamire AM, Ogawa S, Ugurbil K, Rothman D, McCarthy G, Ellermann JM, Hyder F, Rattner Z, Shulman RG (1992) Dynamic mapping of the human visual cortex by high-speed magnetic resonance imaging. Proc Natl Acad Sci USA 89:11069-11073

Britsch PM, Grodd W, Klose U, Ackermann H (1996) Functional mapping of the motor system during voluntary movements with whole brain multislice EPI. Proc ISMRM 4:1865

Buxton RB, Wong EC, Frank LR (1998) Dynamics of blood flow and oxygenation changes during brain activation: the balloon model. Magn Reson Med 39:855-864

Disbrow E, Buonocore M, Antognini J, Carstens E, Rowley H (1998) Somatosensory cortex: a comparison of the response to noxious thermal, mechanical, and electrical stimuli using functional magnetic resonance imaging. Hum Brain Map 6:150-159

Frahm J, Dechent P, Baudewig J, Merboldt KD (2004) Advances in functional MRI of the human brain. Prog Nucl Mag Reson Spectro 44:1-32

Friston KJ, Holmes AP, Worsley KJ, Poline JP, Frith CD, Frackowiak RSJ (1995) Statistical parametric maps in functional imaging: a general linear approach. Hum Brain Map 2:189-210

Gati JS, Menon RS, Ugurbil K, Rutt BK (1997) Experimental determination of the BOLD field strength dependence in vessels and tissue. Magn Reson Med 38:296-302

Haacke EM, Lai S, Reichenbach JR, Kuppusamy K, Hoogenraad FGC, Takeichi H, Lin WL (1997) In vivo measurement of blood oxygen saturation using magnetic resonance imaging: a direct validation of the blood oxygen level-dependent concept in functional brain imaging. Hum Brain Map 5:341-346
Kennedy JM, Zochodne DW (2005) Experimental diabetic neuropathy with spontaneous recovery. Is there irreparable damage? Diabetes 54:830-837

Kim SG, Ashe J, Georgopoulos AP, Merkle H, Ellermann JM, Menon RS, Ogawa S, Ugurbil K (1993) Functional imaging of human motor cortex at high magnetic field. J Neurophysiol 69:297302

Kollias SS, Valavanis A, Golay X, Boesiger P, McKinnon GC (1996) Functional magnetic resonance imaging of cortical activation. Int J Neuroradiol 2:450-472

Kwong KK, Belliveau JW, Chesler DA, Goldberg IE, Weisskoff RM, Poncelet BP, Kennedy DN, Hoppel BE, Cohen MS, Turner $R$ et al (1992) Dynamic magnetic resonance imaging of human brain activity during primary sensory stimulation. Proc Natl Acad Sci USA 89:5675-5679

Larijani B, Zahedi F, Sanjari M, Amini MR, Jalili RB, Adibi H, Vassigh AR (2003) The effect of Ramadan fasting on fasting serum glucose in healthy adults. Med J Malaysia 58:678

Mattson MP, Chan SL, Duan W (2002) Modification of brain aging and neurodegenerative disorders by genes, diet, and behavior. Physiol Rev 82:637-672

Mattson MP, Duan W, Guo Z (2003) Meal size and frequency affect neuronal plasticity and vulnerability to disease: cellular and molecular mechanisms. J Neurochem 84:417-431

McGrath-Hanna NK, Greene DM, Tavernier RJ, Bult-Ito A (2003) Diet and mental health in the arctic: is diet an important risk factor for mental health in circumpolar people? Int J Circumpolar Health 62:228-241

Ogawa S, Lee T, Nayak AS, Glynn P (1990) Oxygenation sensitive contrast in magnetic resonance image of rodent brain at high magnetic fields. Magn Reson Med 14:68-78

Ogawa S, Tank DW, Menon R, Ellermann JM, Kim SG, Merkle H, Ugurbil K (1992) Intrinsic signal changes accompanying sensory stimulation: functional brain mapping with magnetic resonance imaging. Proc Natl Acad Sci USA 89:5951-5955

Raichle ME (1994) Images of the mind: studies with modern imaging techniques. Ann Rev Psychol 45:333-356

Turner R, Le Bihan D, Moonen CT, Despres D, Frank J (1991) Echo-planar time course MRI of cat brain oxygenation changes. Magn Reson Med 22:159-166

Turner R, Jezzard P, Wen H, Kwong KK, Le Bihan D, Zeffiro T, Balaban RS (1993) Functional mapping of the human visual cortex at 4 and 1.5 Tesla using deoxygenation contrast EPI. Magn Reson Med 29:277-279

Wildgruber D, Erb M, Klose U, Grodd W (1997) Sequential activation of supplementary motor area and primary motor cortex during self-paced finger movement in human evaluated by functional MRI. Neurosci Lett 227:161-164

Wildgruber D, Klose U, Grodd W, Erb M, Ackermann H (1996) Dorsolateral prefrontal activation during reversal of automated word orders. Proc ISMRM 4:1866 